\title{
Bidding for the surplus:
}

\section{A non-cooperative approach to the Shapley value ${ }^{*}$}

\author{
David Pérez-Castrillo ${ }^{+}$
}

Universitat Autònoma de Barcelona, Spain

\author{
David Wettstein ${ }^{++}$
}

Ben-Gurion University of the Negev, Israel

\section{W.P. 461.00}

June 2000

\footnotetext{
* We wish to thank Sergiu Hart for several helpful suggestions and conversations, and Ezra Einy, Gerard Hamiache and Roberto Serrano as well as participants in seminars at several universities for helpful comments. Pérez-Castrillo acknowledges financial support from the DGES PB 97-0181 and SGR 96-75. Part of this research was conducted while Wettstein was visiting the Universitat Autònoma de Barcelona, with a grant from the Generalitat de Catalunya, and both authors were visiting the University of Copenhagen, whose financial support is acknowledged.

+ Dep. Economia e Historia Economica and CODE; Universitat Autònoma de Barcelona; Edifici B; 08193 Bellaterra (Barcelona); Spain e-mail: David.Perez@uab.es

++ Corresponding Author: Ben-Gurion University of the Negev; Department of Economics; Monaster Center for Economic Research; Beer-Sheva 84105; Israel

Phone: 9727 - 6472291 Fax 972-7-6472941

e-mail: wettstn@bgumail.bgu.ac.il
} 


\begin{abstract}
We propose a simple mechanism to determine how the surplus generated by cooperation is to be shared in zero-monotonic environments with transferable utility. The mechanism consists of a bidding stage followed by a proposal stage. We show that the subgame perfect equilibrium outcomes of this mechanism coincide with the vector of the Shapley value payoffs. We extend our results to implement the weighted Shapley values. Finally, we generalize our mechanism to handle arbitrary transferable utility environments. The modified mechanism generates an efficient coalition structure, and implements the Shapley values of the super-additive cover of the environment.
\end{abstract}

Keywords: Shapley value, Implementation, Simple mechanism, Coalition formation. Journal of Economic Literature Classification Numbers: C71, C72 


\section{1.- Introduction}

The Shapley value has long been a central solution concept in cooperative game theory. It was introduced in Shapley [24] and was seen as a reasonable way of distributing the gains of cooperation among the players in the game. It is the most studied and widely used single-valued solution concept in cooperative game theory. It has generated various axiomatizations that demonstrate its fairness and consistency properties (see, for instance, Myerson [19], and Hart and Mas-Colell [12], and has been used to impute costs and benefits as in cases of airport runways, phone networks, and political situations. 1

A natural question concerning the Shapley value is whether the agents can reach it through non-cooperative behavior. In other words, is it possible to find a non-cooperative framework that gives rise to the Shapley value as the result of equilibrium behavior? This is part of the Nash program, which tries to provide a non-cooperative foundation for cooperative solution concepts. Several papers have addressed this question in different ways. We will comment on them later in this introduction.

In this paper, we provide a simple non-cooperative game whose outcome always coincides with the Shapley value for zero-monotonic games in characteristic form. We call this game the "bidding mechanism". The basic idea of the bidding mechanism is quite simple. We let one of the players make a proposal to each of the other players, a proposal that will either be accepted by all the other players (in which case the proposal becomes final) or rejected. In the latter case, the proposer is now on his own and the rest of the players play the same game again. If the proposal is accepted, the proposer can form the grand coalition of all the players and collect the value generated in exchange for the proposed payments to the rest of the players.

The question of how the proposer is determined is, of course, central to the design of the bidding mechanism. Indeed, in some games, being the proposer could prove to be beneficial, while in other games it is preferable not to be the proposer. Hence, before the proposal stage is reached, the players will bid to become the proposer, where bids can be positive or negative. ${ }^{2}$ The player with the highest "net bid" (the difference between the 
sum of the bids he makes to the others minus the sum of the bids the others make to him) becomes the proposer and, before proceeding to the proposal stage, pays the bids to the other players. We will show that in the subgame perfect equilibria (SPE) of the bidding mechanism a proposer is determined who will make a proposal that will be accepted by the others. For the proposer, the difference between the value of the grand coalition and the payments and bids paid is her Shapley value. For each of the other players as well, the sum of the bid received plus the accepted proposal is his Shapley value. ${ }^{3}$

Several features of our game make it attractive and different from previous noncooperative approaches to the Shapley value. First, the players obtain the Shapley value in every equilibrium outcome of the game; that is, the implementation is not in expected terms. Also, the game does not imply any a priori randomization that imposes some order on the moves of the players. By adjusting his bids, every player can determine whether he or someone else will be the proposer. Second, the rules of the game are very natural and do not rely on "random" meetings or probabilities that are close to the actual definition of the Shapley value. Hence, the implementation is less "obvious", and provides further support for the use of the Shapley value. Third, the game is finite. Moreover, at equilibrium, it ends in one stage if the game is strictly zero-monotonic (a stage includes three periods of play: bidding, proposing, and accepting or rejecting). Fourth, the strategies played by the players at equilibrium are simple and intuitive. Furthermore, even though the Shapley value plays no role in specifying the rules of the game, the equilibrium strategies are intimately related to the Shapley value itself.

Implementing the Shapley value is not straightforward. For example, Thomson [27] focused on the problems created by strategic behavior and showed that an agent can obtain a better outcome by unilaterally misrepresenting his utility function. Several authors have attempted to realize the Shapley value and overcome such problems.

Gul ([7] and [8]) analyzed a transferable utility economy where random meetings between two agents occur. At each meeting, a randomly chosen party makes an offer to his partner. Acceptance of the offer means that the proposer buys the partner's resources. If the offer is rejected, the meeting dissolves and both agents stay in the market. For strictly convex games, as the time interval between meetings becomes arbitrarily small, 
the expected payoff of each player at an efficient stationary subgame perfect Nash equilibrium (SSPE) converges to his Shapley value. If strict convexity is replaced by strict superadditivity the convergence result holds for those efficient SSPE that entail immediate agreement (Gul [7] and Hart and Levy [11]).

Evans [5] showed that a simplified version of Gul's result follows from the following characterization of the Shapley value. Consider a cooperative game and an associated feasible payoff vector. Assume that players are randomly split into two groups and a representative player is chosen also at random from each group. These two players bargain with each other over how to split the total resources available to all of the players. Following the bargaining process each of the two players has to pay out of his share to the members of his group according to the pre-specified payoff vector. This procedure yields an expected payoff to any player that depends on the initial payoff vector, the random partition mechanism and the solution concept applied to two-person bargaining problems. The initial payoff vector is called consistent if it equals the expected payoff vector. If all partitions are equally likely and the bargaining solution splits the surplus equally, the Shapley payoff vector is the unique consistent payoff vector.

Hart and Mas-Colell [13] proposed a different natural bargaining procedure that supports the Shapley value (as well as the Nash bargaining solution for pure bargaining problems). In their paper, the proposers are also chosen at random but the meetings are multilateral. Agreement requires unanimity. Disagreement puts the proposer in jeopardy, since there is a given probability that he may be removed from the game after a rejection. As the probability of removal becomes small, the SSPE of the procedure yield the Shapley value. ${ }^{4}$ When the probability of removal is one, Hart and Mas-Colell [13] as well as Mas-Colell [18] showed that the expected payoff of any player coincides with his Shapley value. Their mechanism is then the same as our mechanism with the bidding stage replaced by a random determination of the proposer. Krishna and Serrano [15] showed further that for removal probabilities close to one there is a unique SPE of the game proposed by Hart and Mas-Colell [13] that yields the Shapley value payoff vector in expectation. 
In a different spirit, Hart and Moore [14] proposed a game in which agents are lined up and each agent makes an offer to the following agent, where the offer is a contract that may specify what offer this agent has to make to the agent after him. This game implements the Shapley value in SPE. Winter [28] and Dasgupta and Chiu [4] proposed demand commitment games in which each player can either make a demand to the following player or form a coalition satisfying the demands of some of the players preceding him. For strictly convex games, these mechanisms implement the Shapley value in SPE. ${ }^{5}$ In these three works, the implementation is in expected terms since in the first stage of the game the order of the players (or the identity of the first player in Winter, 1994) is randomly chosen, each possible choice having the same probability.

A solution concept closely related to the Shapley value is the weighted Shapley value (Shapley [25]). We also show that a very natural and simple modification of the bidding mechanism implements the weighted Shapley values. ${ }^{6}$

Finally, we generalize the bidding mechanism to deal with all transferable utility environments. In the generalized bidding mechanism, the proposer makes a proposal to each of the other players and, simultaneously, chooses the coalition she wants to form. If all the agents accept the proposal and the coalition, the coalition is formed, and the rest of the players proceed to play the same game among themselves (after having received the proposed payment by the proposer). In the case of rejection, the proposer is on her own and the remaining players play the same game again. In any SPE of this mechanism, the proposer makes a proposal that is accepted. The payoff of the proposer is the difference between the value of the coalition she formed and the payments and bids she made. The payoff to any player in the coalition is the sum of the bid and the proposal accepted. The payoff to players outside the coalition is the sum of the bid, the proposal accepted, and their payment in the continuation game. Hence, the SPE of this mechanism determine a coalition structure and a sharing of the surplus generated under this particular structure. We show that at the SPE of the generalized bidding mechanism the players form an efficient coalition structure. Moreover, the final payments of the players coincide with the Shapley values of the super-additive cover of the game. ${ }^{7}$ 
The paper is organized as follows. Section 2 presents the basic cooperative definitions and Section 3 introduces the bidding mechanism and shows that it implements the Shapley value for zero-monotonic games. In Section 4 we extend our results by implementing the set of weighted Shapley values. In Section 5 we define the generalized bidding mechanism and show that it implements the Shapley value of the super-additive cover of the game. The paper concludes with a brief summary and discussion of further research.

\section{2.- The cooperative model}

Consider a cooperative game in characteristic form $(N, v)$, where $N=\{1, \ldots, n\}$ is the set of players and $v: 2^{N} \rightarrow R$ is a characteristic function satisfying $v(\Phi)=0$ where $\Phi$ is the empty set. For a coalition $S \subseteq N, v(S)$ represents the total payoff that the partners in $S$ can jointly obtain if this coalition is formed. We say that the cooperative game $(N, v)$ is zero-monotonic if $v(S)+v(\{i\}) \leq v(S \cup\{i\})$ for any subset $S \subseteq N$ with $i \notin S$. In a zeromonotonic game there are no negative externalities when a single

player joins a coalition. In sections 2 to 4 of this paper, we restrict our analysis to zeromonotonic games.

We denote by $\phi(N) \in R^{n}$ the Shapley value of the cooperative game $(N, v)$ which is defined by: ${ }^{8}$

$$
\phi_{i}(N)=\sum_{S \subseteq N /\{i\}} \frac{|S| !(n-|S|-1) !}{n !}[v(S \cup\{i\})-v(S)] \text { for all } i \in N,
$$

where $|S|$ denotes the cardinality of the subset $S$. The Shapley value can be interpreted as the expected marginal contribution made by a player to the value of a coalition, where the distributions of coalitions is such that any ordering of the players is equally likely. Also, Shapley [24] characterized the value as the only function that satisfies symmetry, efficiency, a null player axiom, and additivity. 
Given the cooperative game $(N, v)$ and a subset $S \subseteq N$, we define the game $\left(S, v_{S}\right)$ by assigning the value $v_{S}(T) \equiv v(T)$ to every $T \subseteq S$. We write $(S, v)$ instead of $\left(S, v_{S}\right)$ for notational convenience. Similarly, $\phi(S) \in R^{|S|}$ denotes the Shapley value of the game $(S$, $v)$.

\section{3.- The bidding mechanism}

In this section, we design a non-cooperative game, which we call the bidding mechanism. The equilibrium outcomes of this mechanism always coincide with the Shapley value of the cooperative game $(N, v)$ and thus this mechanism implements the Shapley value in subgame perfect equilibria. We propose a way to split the surplus of the cooperation that is based upon the idea that only one of the players will make a proposal to each of the other players. We invoke a notion of consistency in order to determine the outcome of the game if the proposal is rejected. Following a rejection the players other than the proposer play the same game again. Proceeding in this way, the payoff of an agent is sensitive not only to whether or not he is the proposer, but also to the precise identity of the proposer. Hence, in order to provide each player with the same strategic possibilities, each player can, by his bid, directly influence the choice of the proposer. An intriguing feature of the mechanism is that the Shapley value is not the outcome of just one decision, but rather emerges as the cumulative outcome of both the proposal and the bid.

The mechanism is defined recursively. If there is only one player, then he just obtains the value of his stand-alone coalition. It is also useful to describe the bidding mechanism with only two players. It is a three-stage game. First, each player makes a bid to the other. The proposer is then chosen as the player making the highest bid. If the bids are equal the proposer is chosen randomly. The proposer pays the promised bid to her partner. In the second stage, the proposer makes an offer to the other player for him to join her. In the final stage, the player who is not the proposer either accepts or rejects the offer. If he accepts, the grand coalition is formed and the proposer collects the value generated by it while paying the offer to the other player. If the proposal is rejected each 
player is left on his own, and hence each obtains the value of the stand-alone coalition (minus or plus the bid paid previously). Once we know the rules of a two-player bidding mechanism, we can define the mechanism for three players, and so on. Assuming that we know the rules of the bidding mechanism when played by at most $n-1$ players, we now define the game for $n$ players.

First, each of the players makes a bid to each of the other players. To determine the identity of the proposer, we define the "net bid" of a player as the difference between the sum of the bids he makes to the others minus the sum of the bids the others make to him. The net bid of a player tries to measure the difference between the incentives of this player to become the proposer (what he bids) and what the others are ready to pay him for each of them to become the proposer (what the others bid to him). The player with the highest net bid is chosen to be the proposer. If several players make the highest net bid, the proposer is chosen randomly among them. Once "named" a proposer, she proceeds to pay the bids to the other players. Second, the proposer makes an offer to each of the other players to join her. Finally, each of the other players sequentially either accepts or rejects the offer. ${ }^{9}$ The offer is accepted, and all the players join in the grand coalition, only if all of them accept the offer. In this case, the proposer obtains the value of the coalition, paying to the others the promised offers. If the offer is rejected, the proposer is on her own, obtaining the value of her stand-alone coalition (minus the bids she has already paid). ${ }^{10}$ The rest of the players keep their bids and play the same game with $n-1$ players.

It is important to notice that the element of randomness in the determination of the proposer is inconsequential to our proofs. Our results still hold if ties in net bids are broken deterministically as would be the case if the highest indexed player were chosen as the proposer. Randomness is introduced only in order to prevent biased treatment of the participating players. We will return to this issue in the conclusion, when we will discuss possible extensions of our mechanism.

We now describe the bidding mechanism more formally. Suppose first that there is only one player $\{i\}$. Then, this player obtains the value of the stand-alone coalition (i.e., $v(i))$. 
Suppose now that we know the rules of the bidding mechanism when played by at most $n-1$ players. The bidding game for a set of players $N=\{1, \ldots, n\}$ proceeds as follows:

$t=1$ : Each player $i \in N$ makes bids $b_{j}^{i}$ in $R$ for every $j \neq i$. Hence, at this stage, a strategy for player $i$ is a vector $\left(b_{j}^{i}\right)_{j \neq i}$ in $R^{n-1}$.

For each $i \in N$, we let $B^{i}=\sum_{j \neq i} b_{j}^{i}-\sum_{j \neq i} b_{i}^{j}$. Let $\alpha=\operatorname{argmax}_{i}\left(B^{i}\right)$ where, in the case of a nonunique maximizer, $\alpha$ is randomly chosen among the maximizing indices. Once she has been chosen, player $\alpha$ pays $b_{i}^{\alpha}$ to every player $i \neq \alpha$.

$t=2$ : Player $\alpha$ makes an offer $y_{j}^{\alpha}$ in $R$ to every player $j \neq \alpha$. Therefore, at this stage a strategy for player $i$ is a vector $\left(y_{j}^{i}\right)_{j \neq i}$ in $R^{n-1}$ that he will follow if he is chosen to be the proposer.

$t=3$ : The players other than $\alpha$, sequentially, either accept or reject the offer. If a rejection is encountered, we say the offer is rejected. Otherwise, we say the offer is accepted.

If the offer is rejected, all players other than $\alpha$ proceed to play the bidding mechanism where the set of players is $M\{\alpha\}$ and player $\alpha$ obtains the value of her standalone coalition. On the other hand, if the offer is accepted, each player $i \neq \alpha$ receives $y_{i}^{\alpha}$ and player $\alpha$ obtains the value of the grand coalition minus the payments $\sum_{i \neq \alpha} y_{i}^{\alpha}$.

Given that the characteristic function is $v($.$) , the final payment for player \alpha$ in case of rejection is $v(\alpha)-\sum_{i \neq \alpha} b_{i}^{\alpha}$. Final payments for the other players will be the sum of the bid $b^{\alpha}{ }_{i}$ received and the outcome of the mechanism where the players are $\left.M \backslash \alpha\right\}$. In case of acceptance of the proposal, final payment to any player $i$ other than $\alpha$ is given by $y_{i}^{\alpha}+$ $b_{i}^{\alpha}$, whereas player $\alpha$ obtains $v(N)-\sum_{i \neq \alpha} y_{i}^{\alpha}-\sum_{i \neq \alpha} b_{i}^{\alpha}$. 
In order to analyze the outcome of the bidding mechanism, the following wellknown characterization of the Shapley value will be useful. The Shapley value of a player $i$ is the average of the marginal contribution of this player to the grand coalition and his Shapley values in the games where a player different from $i$ has been removed. Or, more formally,

$$
\phi_{i}(N)=\frac{1}{n}(v(N)-v(N \backslash\{i\}))+\frac{1}{n} \sum_{j \neq i} \phi_{i}(N \backslash\{j\}) .
$$

This equation has been previously used by Maschler and Owen [16] and Hart and Mas-Colell [12]. Furthermore, note that it provides a convenient recursive definition of the Shapley value. Defining $\phi_{i}(\{i\})=v(i)$ for every $i$, the previous equation characterizes the Shapley value for every game $(N, v)$.

Theorem 1.- The bidding mechanism implements the Shapley value of the zeromonotonic game $(N, v)$ in SPE.

Proof: The proof proceeds by induction on the number of players $n$. The theorem holds for $k=1$, since for a one-player game, the value of his stand-alone coalition is the Shapley value.

We now assume that the theorem holds for $k=n-1$ and show that it also holds for $k$ $=n$. We take $N=\{1, \ldots, n\}$. We first prove that the Shapley value payoff is indeed an equilibrium outcome. We explicitly construct an SPE that yields the Shapley value as an SPE outcome. Consider the following strategies:

At $t=1$, each player $i, i \in N$, announces $b_{j}^{i}=\phi_{j}(N)-\phi_{j}(N \backslash\{i\})$, for every $j \neq i$.

At $t=2$, player $i, i \in N$, if he is the proposer, offers $y_{j}^{i}=\phi_{j}(N \backslash\{i\})$ to every $j \neq i$.

At $t=3$, player $i, i \in N$, if player $j \neq i$ is the proposer, accepts any offer greater than or equal to $\phi_{i}(M \backslash\{j\})$ and rejects any offer strictly smaller than $\phi_{i}(M \backslash\{j\})$. 
It is clear that these strategies yield the Shapley value for any player who is not the proposer, since $x_{i}^{\alpha}=b_{i}^{\alpha}+y_{i}^{\alpha}=\phi_{i}(N)$, for $i \neq \alpha$. Moreover, given that following the strategies the grand coalition is formed, the proposer also obtains her Shapley value.

We now show that all net bids $B^{i}$ are equal to zero. Following the above mentioned strategies,

$$
B^{i}=\sum_{j \neq i} b_{j}^{i}-\sum_{j \neq i} b_{i}^{j}=\sum_{j \neq i}\left(\phi_{j}(N)-\phi_{j}(N \backslash\{i\})\right)-\sum_{j \neq i}\left(\phi_{i}(N)-\phi_{i}(N \backslash\{j\})\right) .
$$

By the balanced contributions property (see Myerson [19])

$$
\phi_{j}(N)-\phi_{j}(N \backslash\{i\})=\phi_{i}(N)-\phi_{i}(N \backslash\{j\})
$$

and hence $B^{i}=0$.

To check that the previous strategies constitute an SPE note, first, that the strategies at $t=$ 2 and $t=3$ are best responses as long as $v(N)-v(i) \geq \sum_{j \neq i} \phi_{j}(N \backslash\{i\})=v(N \backslash\{i\})$. Indeed, in the case of rejection, a proposer $i$ obtains $v(i)$ and the players $j \neq i$ play the bidding mechanism where $M \backslash\{i\}$ is the set of players; by the induction argument, the outcome of this game is the Shapley value vector $\left.\left(\phi_{j}(M \backslash i\}\right)\right)_{j \neq i}$. Consider now the strategies at $t=1$. If player $i$ increases his total bid $\sum_{j \neq i} b_{j}^{i}$, he will be chosen as the proposer with certainty, but his payoff will decrease. If he decreases his total bid another player will propose, and player $i$ 's payoff would still equal his Shapley value. Finally, any change in his bids that leaves the total bid constant will influence the identity of the proposer but will not alter player $i$ 's payoff.

We now show that any SPE yields the Shapley value. We proceed by a series of claims:

Claim (a). In any SPE, at $t=3$, all players other than the proposer $\alpha$ accept the offer if $\left.y^{\alpha}{ }_{i}>\phi_{i}(M \backslash \alpha\}\right)$ for every player $i \neq \alpha$. Moreover, if $y_{i}^{\alpha}<\phi_{i}(M\{\alpha\})$ for at least some $i \neq$ $\alpha$, then the offer is rejected. 
Note that in the case of rejection, by the induction argument the payoff to a player $i \neq \alpha$ is $\left.\phi_{i}(M \backslash \alpha\}\right)$. We denote the last player that has to decide whether to accept or reject the offer, at $t=3$, by $\beta$. If the game reaches player $\beta$, i.e., there has been no previous rejection, his optimal strategy involves accepting any offer higher than $\phi_{\beta}(N\{\alpha\})$ and rejecting any offer lower than $\left.\phi_{\beta}(M \backslash \alpha\}\right)$. The second to last player (denoted by $\beta-1$ ) anticipates the reaction of player $\beta$. Hence, if $y_{\beta-1}^{\alpha}>\phi_{\beta-1}(M\{\alpha\})$ and $\left.y_{\beta}^{\alpha}>\phi_{\beta}(M \backslash \alpha\}\right)$, and the game reaches player $\beta-1$, he will accept the offer. If $\left.y_{\beta-1}^{\alpha}<\phi_{\beta-1}(M \backslash \alpha\}\right)$ and $y_{\beta}^{\alpha}$ $>\phi_{\beta}(M\{\alpha\})$, he will reject the offer. If $y_{\beta}^{\alpha}<\phi_{\beta}(M\{\alpha\})$, player $\beta-1$ is indifferent to accepting or rejecting any offer $y_{\beta-1}^{\alpha}$, since he knows that player $\beta$ is bound to reject the offer should the game reach him. In any case, the offer is rejected. We can go backwards using the same argument to prove claim (a).

Claim (b). If $v(N)>v(N \backslash \alpha\})+v(\alpha)$, the only SPE of the game that starts at $t=2$ is the following: At $t=2$, player $\alpha$ offers $y^{\alpha}{ }_{i}=\phi_{i}(M\{\alpha\})$ to all $i \neq \alpha$; at $t=3$, every player $i$ $\neq \alpha$ accepts any offer $y_{i}^{\alpha} \geq \phi_{i}(M\{\alpha\})$ and rejects the offer otherwise.

If $v(N)=v(M\{\alpha\})+v(\alpha)$ there exist SPE in addition to the previous one. Any set of strategies where, at $t=2$, the proposer offers $y_{j}^{\alpha} \leq \phi_{j}(M\{\alpha\})$ to a particular player $j \neq$ $\alpha$ and, at $t=3$, the player $j$ rejects any offer $\left.y_{j}^{\alpha} \leq \phi_{j}(M \backslash \alpha\}\right)$, also constitutes an SPE.

In all the SPE of this subgame, the final payoffs to players $\alpha$ and $i \neq \alpha$ are $v(N)-v(N \backslash\{\alpha\})-\sum_{j \neq \alpha} b_{j}^{\alpha}$ and $\phi_{i}(N \backslash\{\alpha\})+b_{i}^{\alpha}$, respectively.

It is easy to see that the proposed strategies constitute an SPE. Suppose now that $v(N)>$ $v(N \backslash\{\alpha\})+v(\alpha)$. In that case, rejection of the offers made by player $\alpha$ cannot be part of an SPE. In such a case, player $\alpha$ receives $v(\alpha)$. She can improve her payoff by offering $\left.\phi_{i}(M \backslash \alpha\}\right)+\varepsilon /(n-1)$ to every $i \neq \alpha$, with $\varepsilon<v(N)-v(N\{\alpha\})-v(\alpha)$ and $\varepsilon>0$ so that her offers are accepted (by (a)). Therefore, an SPE requires acceptance of the proposal. This implies $y_{i}^{\alpha} \geq \phi_{i}(M\{\alpha\})$ for all $i \neq \alpha$. However, an offer such that $y_{j}^{\alpha}>\phi_{j}(M\{\alpha\})$ for some $j \neq \alpha$ cannot be part of an SPE, since $\alpha$ could still offer $\phi_{i}(M\{\alpha\})+\varepsilon /(n-1)$ to every $i \neq$ $\alpha$, with $\varepsilon<y_{j}^{\alpha}-\phi_{j}(M\{\alpha\})$ and $\varepsilon>0$. These offers are accepted and $\alpha$ 's payoff increases. 
Hence, $\left.y_{i}^{\alpha}=\phi_{i}(M \backslash \alpha\}\right)$ for all $i \neq \alpha$ at any SPE. Finally, acceptance of the proposals implies that, at $t=3$, every agent $i \neq \alpha$ accepts an offer if $y^{\alpha}{ }_{i} \geq \phi_{i}(M\{\alpha\})$.

If $v(N)=v(M \backslash \alpha\})+v(\alpha)$, then the proposer has to offer at least $\sum_{j \neq \alpha} \phi_{j}(N \backslash\{\alpha\})=$ $v(N \backslash\{\alpha\})$ for the offer to be accepted by every other player. By the same argument as in the previous case, every equilibrium in which the offer is accepted necessarily involves a proposal of exactly $\phi_{j}(N \backslash\{\alpha\})$ for every $j \neq \alpha$. Given that the proposer obtains $v(\alpha)$ in case of rejection, any offer that leads to a rejection would be an SPE as well.

Notice that following the first strategies, the offer is accepted and the grand coalition is formed, while the second strategies imply that the proposer is left on her own. Given that the last strategies are SPE only when $v(N)=v(M\{\alpha\})+v(\alpha)$, it is easy to check that the final payoffs are those stated in the claim.

Claim (c). In any SPE, $B^{i}=B^{j}$ for all $i$ and $j$ and hence $B^{i}=0$ for all $i$ in $N$.

Denote $\Omega=\left\{i \in N \mid B^{i}=\operatorname{Max}_{j}\left(B^{j}\right)\right\}$. If $\Omega=N$ the claim is satisfied since $\sum_{i \in N} B^{i}=0$.

Otherwise, we can show that any player $i$ in $\Omega$ can change his bids so as to decrease the sum of payments in case he wins. Furthermore, these changes can be made without altering the set $\Omega$. Hence, he maintains the same probability of winning, and obtains a higher expected payoff. Take some player $j \notin \Omega$. Let player $i \in \Omega$ change his strategy by announcing: $b^{{ }^{i}}{ }_{k}=b_{k}^{i}+\delta$ for all $k \in \Omega$ and $k \neq i ; b^{{ }^{i}}{ }_{j}=b_{j}^{i}-|\Omega| \delta$; and $b^{{ }^{i}}{ }_{l}=b_{l}{ }_{l}$ for all $l \notin \Omega$ and $l \neq j$. The new net bids are: $B^{\prime i}=B^{i}-\delta ; B^{\prime k}=B^{k}-\delta$ for all $k \in \Omega$ and $k \neq i ; B^{\prime j}=B^{j}+$ $|\Omega| \delta$ and $B^{\prime l}=B^{l}$ for all $l \notin \Omega$ and $l \neq j$. If $\delta$ is small enough, so that $B^{j}+|\Omega| \delta<B^{i}-$ $\delta$ (remember that $B^{j}<B^{i}$ ), then $B^{\prime} l<B^{\prime i}=B^{\prime k}$ for all $l \notin \Omega$ (including $j$ ) and for all $k \in \Omega$. Therefore, $\Omega$ does not change. However, $\sum_{h \neq i} b_{h}^{i}-\delta<\sum_{h \neq i} b_{h}^{i}$.

Claim (d). In any SPE, each player's payoff is the same regardless of who is chosen as the proposer. 
We already know that all the bids $B^{i}$ are the same. If player $i$ would strictly prefer to be the proposer, he could improve his payoff by slightly increasing one of his bids $b_{j}^{i}$. Similarly, if player $i$ would strictly prefer that some other player $j$ were the proposer, he could improve his payoff by decreasing $b_{j}^{i}$. The fact that player $i$ does not do so in equilibrium means that he is indifferent to the proposer's identity.

Claim (e). In any SPE, the final payment received by each of the players coincides with his Shapley value.

Note first that, if player $i$ is the proposer, his final payoff is given by: $x_{i}^{i}=v(N)-v(N \backslash\{i\})-\sum_{j \neq i} b_{j}^{i}$. On the other hand, if player $j \neq i$ is the proposer, the final payoff of player $i$ is given by: $x_{i}^{j}=\phi_{i}(N \backslash\{j\})+b_{i}^{j}$. Therefore, the sum of payoffs to player $i$ over all possible choices of the proposer is given by:

$$
\begin{aligned}
& \sum_{j} x_{i}^{j}=\left(v(N)-v(N \backslash\{i\})-\sum_{j \neq i} b_{j}^{i}\right)+\sum_{j \neq i}\left(\phi_{i}(N \backslash\{j\})+b_{i}^{j}\right)= \\
& v(N)-v\left(N \backslash\{i\}+\sum_{j \neq i} \phi_{i}(N \backslash\{j\})-B^{i}=v(N)-v(N \backslash\{i\})+\sum_{j \neq i} \phi_{i}(N \backslash\{j\})=n \phi_{i}(N),\right.
\end{aligned}
$$

Moreover, since player $i$ is indifferent to all possible choices of the proposer, we have $x_{i}^{j}$ $=x_{i}{ }_{i}$ for all $j, k$. Therefore $x_{i}^{j}=\phi_{i}(N)$ for all $j$ in $N$. Q. E. D.

The theorem, in addition to showing that the mechanism indeed realizes the Shapley value, provides us with the explicit form of the equilibrium strategies. The ease by which these strategies can be computed adds further credibility to our results and helps in the actual implementation of the mechanism. At equilibrium, the bid of player $i$ to player $j$ is $\phi_{j}(N)-\phi_{j}(N \backslash\{i\})$. The balanced contributions property (see Myerson [19]) implies that the bid can also be expressed as $\phi_{i}(N)-\phi_{i}(N \backslash\{j\})$, which is the contribution of player $j$ to the Shapley value of player $i$. In particular, the bids are symmetric: player $i$ bids for $j$ just as much as player $j$ bids for $i$. Furthermore, the determination of the offers is also simple. If player $i$ is the proposer, he offers $\phi_{j}(M\{i\})$ to any other player $j$. The offer reflects the outside options of the players other than the proposer. Due to the recursive 
nature of our mechanism, these options are given by their Shapley value in the game without the proposer. Finally, notice that if the game is strictly zero-monotonic ${ }^{11}$ not only is the equilibrium outcome unique, but the equilibrium strategies are unique as well. This eliminates problems of coordination among the players.

As we pointed out in the informal description of the mechanism, Theorem 1 holds if proposer $\alpha$ obtains a payment $u(\alpha)$ lower than $v(\alpha)$ in case her offer is rejected. This is a more reasonable assumption in those circumstances in which the technology is not replicable. In such a case $v(S)$ represents the payoff to the partners in $S$ only if they have access to the technology. If $u(i)<v(i)$ for every $i$ in $N$, then the equilibrium strategies are unique even if the game is zero-monotonic and not strictly zero-monotonic.

A further advantage of the mechanism is that it is finite in contrast to the infinite horizon mechanisms that implement the Shapley value in stationary SPE. Moreover, at the proposed equilibrium strategies, only the first stage of the game is played, with the proposal made by the chosen proposer accepted by the other players.

We can modify our mechanism by replacing the bidding stage with a random selection of the proposer. Once the proposer is determined, the game proceeds similarly to our mechanism with the only difference being that in case of rejection the new proposer is randomly selected from the remaining players. This modified mechanism coincides with the Mas-Colell [18] and Hart and Mas-Colell [13] (with removal probability equal to one) construction. In this mechanism, however, the equilibrium payoffs yield the Shapley value in expected terms only.

\section{4.- Implementation of the weighted Shapley values}

The weighted Shapley value emerges out of considering non-symmetric divisions of the surplus. It is defined in Shapley [25] by stipulating an exogenously given system of weights $w \in R^{n}{ }_{++}$. Each unanimity game is assigned a value by having agent $i$ receive the

share $w^{i} / \sum_{j \in N} w^{j}$ of the unit. The $w$-weighted Shapley value of a game is defined as the 
linear extension of this operator to the game. We denote by $\phi_{w i}(N)$ the $w$-weighted Shapley value of player $i$ in the cooperative game $(N, v)$.

A convenient way to express the weighted Shapley value is through the weighted potential function $P_{w}(N)$ defined in Hart and Mas-Colell [12]. ${ }^{12}$ The $w$-weighted potential $P_{w}(N)$ is the unique function from the set of games into $R$ that satisfies $P_{w}(\Phi)=0$ and $\sum_{i \in N} w^{i} D^{i} P_{w}(N)=v(N)$, where $D^{i} P_{w}(N)=P_{w}(N)-P_{w}(M\{i\})$. This function satisfies: $w^{i} D^{i} P_{w}(N)=\phi_{w i}(N)$. Furthermore,

$$
P_{w}(N)=\frac{1}{\sum_{j \in N} w^{j}}\left[v(N)+\sum_{j \in N} w^{j} P_{w}(N \backslash\{j\})\right]
$$

The weighted Shapley value, as the Shapley value, can be calculated using a recursive procedure. The role played by this formula in the proof of Theorem 2 is similar to the role played by the recursive formula characterizing the Shapley value in the proof of Theorem 1:

Lemma 1.- The weighted Shapley value of player $i$ satisfies the equality:

$$
\phi_{w i}(N)=\frac{1}{\sum_{j \in N} w^{j}}\left[w^{i}(v(N)-v(N \backslash\{i\}))+\sum_{j \neq i} w^{j} \phi_{w i}(N \backslash\{j\})\right] .
$$

Proof.- The weighted Shapley value of player $i$ satisfies:

$$
\begin{aligned}
& \phi_{w i}(N)=w^{i}\left[P_{w}(N)-P_{w}(N \backslash\{i\})\right]=w^{i} \frac{1}{\sum_{j \in N} w^{j}}\left[v(N)+\sum_{j \in N} w^{j} P_{w}(N \backslash\{j\})-\sum_{j \in N} w^{j} P_{w}(N \backslash\{i\})\right]= \\
& \frac{1}{\sum_{j \in N} w^{j}}\left[w^{i} v(N)+\sum_{j \neq i} w^{j}\left(w^{i} P_{w}(N \backslash\{j\})-w^{i} P_{w}(N \backslash\{i, j\})-w^{i} P_{w}(N \backslash\{i\})+w^{i} P_{w}(N \backslash\{i, j\})\right)\right]= \\
& \frac{1}{\sum_{j \in N} w^{j}}\left[w^{i} v(N)+\sum_{j \neq i} w^{j} \phi_{w i}(N \backslash\{j\})-w^{i} \sum_{j \neq i} \phi_{w j}(N \backslash\{i\})\right]=
\end{aligned}
$$


$\frac{1}{\sum_{j \in N} w^{j}}\left[w^{i}(v(N)-v(N \backslash\{i\}))+\sum_{j \neq i} w^{j} \phi_{w i}(N \backslash\{j\})\right]$ Q.E.D

We will now indicate how to modify our original bidding mechanism in order to obtain as an equilibrium outcome any weighted Shapley value. The only difference is in the construction of the weighted net bids $B_{w}{ }^{i}$. The determination of net bids incorporates the vector of weights $w \in R^{n}{ }_{++}$by having $B_{w}{ }^{i}=\sum_{j \neq i} w^{i} b_{j}^{i}-\sum_{j \neq i} w^{j} b_{i}^{j}$. Other than that change, the weighted bidding mechanism proceeds like the bidding mechanism. Intuitively we weigh each bid differently, according to the exogenously given weight of the person making the bid.

Theorem 2.- The weighted bidding mechanism implements the weighted Shapley value of the zero-monotonic game $(N, v)$ in SPE.

The proof of Theorem 2 is similar to the proof of Theorem 1.

Finally, note that we can implement the weighted Shapley value in expected terms by using a simpler mechanism (similar to the Mas-Colell [18] and Hart and Mas-Colell [13], construction for the Shapley value). Given a system of weights $w \in R^{n}{ }_{++}$, we replace the bidding stage by a random choice of the proposer, where the probability of player $i$ to be chosen as the proposer equals $w^{i} / \sum_{j \in N} w^{j}$ (rather than $1 / n$ ).

\section{5.- General transferable utility games and the formation of coalitions}

The only requirement we have imposed so far on the cooperative environment is that of zero-monotonicity. Zero-monotonic environments might still violate super-additivity. Therefore the (weighted) bidding mechanism implements the (weighted) Shapley value even in some non super-additive settings. This result however is not entirely satisfactory since the outcome while coinciding with the Shapley value might not be "really" efficient. The sum of payments would indeed equal $v(N)$, yet $v(N)$ might not be the maximal payoff the players could obtain. Note that in non super-additive environments it 
might be possible for the players to obtain a sum of payments that exceeds $v(N)$ by splitting up into two or more coalitions.

One way to resolve this difficulty might be to consider the super-additive cover of the environment. If we apply our mechanism to the super-additive cover of the environment rather than to the original environment, the equilibria outcomes would coincide with the Shapley value of the super-additive cover. A possible disadvantage of this approach is that a player (the proposer) is able to collect rents from a coalition of which she is not an active member. In other words, a player can act as a "principal" for a coalition formed by other players. ${ }^{13}$

One way to avoid the use of "principals" is to modify our mechanism. The new generalized bidding mechanism would generate a coalition structure in which proposers would receive (when there is no rejection) just the value of the coalition to which they belong. In this mechanism the players would not only share the surplus but would also form coalitions in a sequential way. We show that at any SPE, the coalitions formed will constitute an efficient coalition structure and the final payments of the players will coincide with the Shapley value of the super-additive cover of the environment.

Before proceeding with the formal description of the generalized bidding mechanism we introduce the following notation. The super-additive (SA) cover of a cooperative game in characteristic form $(N, v)$, is denoted by $(N, V)$. The value $V(S)$, for $S \subseteq N$, is defined by: $V(S)=\operatorname{Max}_{\pi}\left\{\sum_{S \in \pi} v(S) \mid \pi\right.$ is a partition of $\left.S\right\}$.

We denote the Shapley value of player $i$ in the SA cover of $(N, v)$ by $\Theta_{i}(N)$, and similarly for the values $\Theta_{i}(S)$ of subsets $S$ of $N$.

We know that: $\quad \Theta_{i}(N)=\frac{1}{n}(V(N)-V(N \backslash\{i\}))+\frac{1}{n} \sum_{j \neq i} \Theta_{i}(N \backslash\{j\})$.

A partition $\pi$ such that $V(N)=\sum_{S \in \pi} v(S)$ is called an efficient partition for $N$. 
The generalized bidding mechanism $(\mathrm{GBM})$ is similar to the bidding mechanism. The only difference is that in the GBM, the proposer, in addition to offering a vector of payments to all the other players, also chooses a coalition she wants to form and be a member of. Hence, an offer by the proposer consists of a payments vector and a coalition. The offer is accepted if all the other players agree. In case of acceptance the coalition is formed, the proposer collects the value of that coalition and the players outside the coalition proceed to play the same game again among themselves. In the case of rejection all the players other than the proposer play the same game again.

Formally, if there is only one player $\{i\}$, she obtains the value of the stand-alone coalition. Given the rules of the game when played by at most $n-1$ players, the game for $N=\{1, \ldots, n\}$ players proceeds as follows:

$$
t=1 \text { : Each player } i \in N \text { makes bids } b_{j}^{i} \text { in } R \text { for every } j \neq i \text {. }
$$

Player $\alpha$ is chosen as in the bidding mechanism. She pays $b^{\alpha}$ to every player $i \neq \alpha$.

$t=2$ : Player $\alpha$ chooses a coalition $S_{\alpha}$ with $\alpha \in S_{\alpha}$ and makes an offer $y^{\alpha}{ }_{i}$ in $R$ to every player $i \neq \alpha$.

$t=3$ : The players other than $\alpha$, sequentially, either accept or reject the offer. If an agent rejects it, then the offer is rejected. Otherwise, the offer is accepted.

If the offer is accepted, each player $i \neq \alpha$ receives $y_{i}^{\alpha}$ and player $\alpha$ receives the value of the coalition $S_{\alpha}$ minus the payments $\sum_{i \neq \alpha} y_{i}^{\alpha}$. After this, players in $M S_{\alpha}$ proceed to play the GBM again among themselves. (Therefore, final payment to a player $i \in S_{\alpha} \backslash\{\alpha\}$ is $y_{i}^{\alpha}+$ $b_{i}^{\alpha}$, player $\alpha$ receives $v\left(S_{\alpha}\right)-\sum_{i \neq \alpha} y_{i}^{\alpha}-\sum_{i \neq \alpha} b_{i}^{\alpha}$, and the final payment for a player $i \in M S_{\alpha}$ will be the sum of the bid $b_{i}^{\alpha}$, the offer $y_{i}^{\alpha}$, and the outcome of the GBM where the players are $N S_{\alpha .}$.) On the other hand, if the offer is rejected, all players other than $\alpha$ proceed to play the GBM where the set of players is $N \backslash \alpha \alpha$ and player $\alpha$ receives the value of her stand-alone coalition. 
Theorem 3.- The generalized bidding mechanism implements the Shapley value of the SA cover of the game $(N, v)$.

Proof: The arguments, in part, are very similar to those used in Theorem 1, thus we emphasize just the new features of this proof and otherwise rely on the reasoning employed in Theorem 1.

It is easy to see that the theorem holds for $k=1$. We assume that it holds for $k=n-1$ and then consider the following strategies:

$$
\text { At } t=1 \text {, each player } i, i \in N \text {, announces } b_{j}^{i}=\Theta_{j}(N)-\Theta_{j}(M\{i\}) \text {, for every } j \neq i \text {. }
$$

At $t=2$, player $i, i \in N$, if she is the proposer, chooses a coalition $S_{i}$ such that $S_{i} \in$ $\operatorname{Argmax}_{S \subseteq N}\{v(S)+V(M S) \mid i$ in $S\}$ and offers $y_{j}^{i}=\Theta_{j}(N \backslash\{i\})$ to every $j \in S_{i} \backslash\{i\}$ and $y_{j}^{i}=\Theta_{j}(N \backslash\{i\})-\Theta_{j}\left(N \backslash S_{i}\right)$ to every $j \notin S_{i}$.

At $t=3$, player $i, i \in N$, if player $j \neq i$ is the proposer and $i \in S_{j}$, accepts any offer greater than or equal to $\Theta_{i}(M\{j\})$ and rejects it otherwise. If player $j \neq i$ is the proposer and $i \notin S_{j}$, player $i$ accepts any offer greater than or equal to $\left.\Theta_{i}(M \backslash j\}\right)-\Theta_{i}\left(M S_{j}\right)$ and rejects it otherwise.

Following these strategies, the proposer selects a coalition $S_{\alpha}$ that is part of an efficient partition. ${ }^{14}$ Also, the induction argument ensures that, in the game that follows among the players in $M S_{\alpha}$, player $i \notin S_{\alpha}$ will obtain $\Theta_{i}\left(M S_{\alpha}\right)$. It is then easy to see that the previous strategies yield $\Theta_{i}(N)$ to any player $i$.

To prove that the previous strategies constitute an SPE, note, first, that the strategy at $t=$ 3 is a best response for any player different from the proposer by the same argument we used in Theorem 1. At $t=2$, given the rejection criteria used by the other players, if player $i$ is the proposer, she chooses a subset $S_{i}$ that maximizes:

$$
\left.v\left(S_{i}\right)-\sum_{j \in S_{i} \backslash\{i\}} \Theta_{j}(N \backslash\{i\})-\sum_{j \notin S_{i}}\left[\Theta_{j}(N \backslash\{i\})-\Theta_{j}\left(N \backslash S_{i}\right)\right]=v\left(S_{i}\right)+V\left(N S_{i}\right)-\mathrm{V}(M \backslash i\}\right) .
$$


Therefore, the proposed strategy is optimal. Finally, an argument similar to the one in the proof of Theorem 1 demonstrates the optimality of the strategies at $t=1$.

To show that any SPE yields the Shapley value, we proceed by a series of claims. We state the claims without proof, since they are similar to those in Theorem 1. To simplify notation, we denote the "effective offer" to player $i \neq \alpha$ in stage 2 , when player $\alpha$ is the proposer by $z^{\alpha}{ }_{i}$, and define it as $z^{\alpha}{ }_{i}=y_{i}^{\alpha}$ if $i \in S_{\alpha} \backslash\{\alpha\}$ and $z_{i}^{\alpha}=y_{i}^{\alpha}+\Theta_{i}\left(M S_{\alpha}\right)$ if $i$ $\notin S_{\alpha}$. By the induction argument, the effective offer is the total payment (without taking into account the bid already received) that a player will receive (at equilibrium) if the offer is accepted.

Claim (a). In any SPE, at $t=3$, any player $j \neq \alpha$ accepts the offer if $z_{j}^{\alpha}$ is strictly greater than $\left.\Theta_{i}(M \backslash \alpha\}\right)$ for every player $i \neq \alpha$. Moreover, if $z^{\alpha}{ }_{i}<\Theta_{i}(M\{\alpha\})$ for at least some $i \neq \alpha$, then the offer is rejected.

Claim (b). If the coalition $\{\alpha\}$ is not part of any efficient partition, then in any SPE of the game that starts at $t=2, \alpha$ will choose a coalition $S_{\alpha}$ that is part of an efficient partition. Furthermore, player $\alpha$ will announce offers such that $z_{i}^{\alpha}=$ $\Theta_{i}(M\{\alpha\})$ for any player $i \neq \alpha$. Finally, at $t=3$, every player $i \neq \alpha$ accepts any offer such that $\left.z_{i}^{\alpha} \geq \Theta_{i}(M \backslash \alpha\}\right)$.

If the coalition $\{\alpha\}$ is part of any efficient partition, there exist other equilibria in addition to the previous ones. Any set of strategies where, at $t=2$, the proposer makes offers such that $z_{j}^{\alpha} \leq \Theta_{j}(M\{\alpha\})$ to a particular player $j \neq \alpha$ and, at $t=3$, the player $j$ rejects any effective offer less than or equal to $\Theta_{j}(M\{\alpha\})$, also constitute an SPE.

In all the SPE of this subgame, the payoffs (taking into account the continuation of the game after $S_{\alpha}$ has been formed) to players $\alpha$ and $i \neq \alpha$ are $\left.V(N)-V(N \backslash \alpha\}\right)-$ $\sum_{j \neq \alpha} b_{j}^{\alpha}$ and $\Theta_{i}(N \backslash\{\alpha\})+b_{i}^{\alpha}$, respectively.

(Notice that following both types of strategies an efficient partition is formed.) 
Claim (c). In any SPE, $B^{i}=0$ for all $i$ in $N$.

Claim (d). In any SPE, each player's payoff is the same regardless of who is chosen as the proposer.

Claim (e). In any SPE, the final payment received by each of the players coincides with his Shapley value in the SA cover.

Q. E. D.

Theorem 3 shows that when facing environments where forming the grand coalition might not be efficient, it is possible to employ a generalized version of our original bidding mechanism that allows both that an efficient partition can be formed and that the surplus can be shared in a "reasonable" way. If the game is super-additive, the generalized version yields the same outcome as the bidding mechanism. It is however important to notice that, if the game is not super-additive but the grand coalition is efficient, this coalition is formed under both mechanisms although the sharing of the surplus will be different. The reason is that the Shapley value of the super-additive cover usually does not coincide with the Shapley value of the game if the game is not superadditive.

Our GBM provides support for the use of the Shapley value of the SA cover as the generalization of the Shapley value for games in which it is efficient to form coalition structures which are different from the grand coalition. The GBM implements the Shapley value of the SA cover by simultaneously providing a bidding and coalition formation game. To the best of our knowledge, this is the first paper that supports this solution concept. Aumann and Dréze [1] study games with a (given) coalition structure and define a value that assigns to each player his Shapley value in the coalition he belongs to. Under this concept, the payoff to any player does not depend upon his contribution to coalitions other than his coalition. The Shapley value of the super-additive cover takes into account not only the contribution of a player to the coalition he belongs to in an efficient structure, but also his potential contribution to any other coalition. ${ }^{15}$ 


\section{6.- Conclusion}

The object of this paper was to construct a simple non-cooperative mechanism to realize a sharing of the surplus in a cooperative environment. The mechanism we use basically consists of two distinct stages of play: a bidding stage, at the end of which a winner is determined, followed by a proposal stage where the winner offers a sharing of the surplus. In the case where the proposal is rejected, the same game is played again by the players except for the proposer. We show that the payoff outcome of the subgame perfect equilibria of this game always coincides with the Shapley value of the game. Moreover, the strategies played by the players at equilibrium are simple and natural. We also showed that a natural modification of the mechanism implements the weighted Shapley value. Finally, we have introduced a simple generalization of the bidding mechanism that handles situations where the grand coalition might not be efficient. By playing the game, the players form, at equilibrium, an efficient coalition structure and share the surplus according to the Shapley value of the super-additive cover of the environment.

These mechanisms provide strong support for applying the Shapley value to situations where cooperation is needed to obtain an efficient outcome. It might be also used for a variety of cost allocation, revenue sharing, or partnership dissolution problems.

The general approach taken in this paper may yield ways to provide non-cooperative foundations for other cooperative solution concepts for transferable utility games or for cost-sharing methods. However, the extension of our approach to non-transferable utility games is problematic. There exist several extensions of the Shapley value to nontransferable utility games proposed by Harsanyi [9], Shapley [26], and Maschler and Owen ([16],[17]). Dagan and Serrano [3] have shown that randomness is a necessary component in a mechanism designed to implement any of these extensions. Since the element of randomness in our mechanism (i. e., the tie-breaking rule) is inconsequential to proving our results, it seems that the approach taken in this paper would fail to implement the existing extensions of the Shapley value. 


\section{References}

1. R. Aumann and J. Dréze, Cooperative games with coalition structure, Int. J. Game Theory 3 (1974), 217-237.

2. V. P. Crawford, A procedure for generating Pareto-efficient egalitarian-equivalent allocations, Econometrica 47 (1979), 49-60.

3. N. Dagan and R. Serrano, Invariance and randomness in the Nash program for coalitional games, Econ. Letters 58 (1998), 43-49.

4. A. Dasgupta and Y. S. Chiu, On implementation via demand commitment games, Int. J. Game Theory 27 (1996), 161-189.

5. R. A. Evans, Value, consistency, and random coalition formation, Games Econ. Behav. 12 (1996), 68-80, doi:10.1006/game.1966.0005.

6. R. A. Evans, Coalitional bargaining with competition to make offers, Games Econ. Behav. 19 (1997), 211-220.

7. F. Gul, Bargaining foundations of Shapley value, Econometrica 57 (1989), 81-95.

8. F. Gul, Efficiency and immediate agreement: A reply to Hart and Levy, Econometrica, 67 (1999), 913-917.

9. J. C. Harsanyi, A simplified bargaining model for the $n$-person cooperative game," Int. Econ. Rev. 4 (1963), 194-220.

10. S. Hart and M. Kurz, Endogenous formation of coalitions, Econometrica 51 (1983), 1047-1064.

11. S. Hart and Z. Levy, Efficiency does not imply immediate agreement, Econometrica 67 (1999), 909-912.

12. S. Hart and A. Mas-Colell, Potential, value, and consistency, Econometrica 57 (1989), 589-614. 
13. S. Hart and A. Mas-Colell, Bargaining and value, Econometrica 64 (1996), 357-380.

14. O. Hart and J. Moore, Property rights and the nature of the firm, J. Polit. Econ. 98 (1990), 1119-1158.

15. V. Krishna and R. Serrano, Perfect equilibria of a model of N-person noncooperative bargaining, Int. J. Game Theory 24 (1995), 259-272.

16. M. Maschler and G. Owen, The consistent Shapley value for hyperplane games, Int. J. Game Theory 18 (1989), 389-407.

17. M. Maschler and G. Owen, The consistent Shapley value for games without side payments, in "Rational interaction" (R. Selten, ed.), pp. 5-12, Springer-Verlag, New York, 1992.

18. A. Mas-Colell, Algunos comentarios sobre la teoría cooperativa de los juegos, Cuadernos Económicos de ICE 40 (1988), 143-161.

19. R. B. Myerson, Conference structures and fair allocation rules, Int. J. Game Theory 9 (1980), 169-182.

20. G. Owen, Values of games with a priori unions, in "Essays in Mathematical Economics and Game Theory" (R. Hein and O. Moeschlin, Eds.), pp. 76-88, Springer-Verlag, New York, 1977.

21. D. Pérez-Castrillo, Cooperative outcomes through non-cooperative games, Games Econ. Behav. 7 (1994), 428-440.

22. D. Pérez-Castrillo and D. Wettstein, Implementation of bargaining sets via simple mechanisms, Games Econ. Behav. 31 (2000), 106-120.

23. A. E. Roth, Introduction to the Shapley value, in "The Shapley Value: Essays in Honor of Lloyd S. Shapley" (A.E. Roth Ed.), pp. 1-27, Cambridge University Press, Cambridge, 1988. 
24. L. S. Shapley, A value for n-person games, in "Contributions to the Theory of Games II (Ann. Math. Studies, 28)" (H.W. Kuhn and A.W. Tucker Eds.), pp. 307317, Princeton University Press, Princeton, 1953.

25. L. S. Shapley, "Additive and non-additive set functions," Ph.D. thesis, Princeton University, Princeton, 1953.

26. L. S. Shapley, Utility comparison and the theory of games, in "La Décision" (Editions du CNRS), pp. 251-263, Paris, 1969.

27. W. Thomson, The manipulability of the Shapley value, Int. J. Game Theory 17 (1988), 101-127.

28. E. Winter, The demand commitment bargaining and snowballing cooperation, Econ. Theory 4 (1994), 255-273. 
${ }^{1}$ For a nice introduction to the Shapley value and, in particular, its applications, see, for example Roth [23].

2 Crawford [2] also made use of a bidding stage in a procedure to generate Pareto-efficient egalitarianequivalent allocations. The discrete time non-cooperative coalitional bargaining game proposed by Evans [6] to implement the core in subgame perfect equilibria also introduced simple bidding by the players for the right to make an offer.

${ }^{3}$ The equilibrium strategies are unique if the game is strictly zero-monotonic. Otherwise, there might be other equilibria in addition to this one, but they still yield the Shapley value.

${ }^{4}$ They also show that for NTU games, the limit of the SSPE (as the probability of removal becomes small) is the consistent value, a solution concept that was introduced by Maschler and Owen ([16], [17]).

${ }^{5}$ Winter [28] also required either subgame consistency or strategic equilibria. Dasgupta and Chiu [4] also developed an implementation for general games in characteristic form if there is an (external) planner who is able to impose a system of transfers and taxes.

${ }^{6}$ Hart and Mas-Colell [12] also extended their results to weighted Shapley values.

${ }^{7}$ If the game is super-additive, the grand coalition is an efficient structure and the Shapley value of the super-additive cover coincides with the Shapley value. Therefore, the final SPE outcome of the generalized bidding mechanism is the same as the final SPE outcome of the bidding mechanism.

${ }^{8}$ We use $\phi(N)$ instead of $\phi(N, v)$ for notational simplicity.

${ }^{9}$ Note that the actual sequence of players is inconsequential. The fact that players respond in sequence rather than simultaneously is crucial for ruling out "bad" equilibria. In bad equilibria, there are several players rejecting the proposal since whenever there is at least one rejection, a rejection by any other player is optimal (the proposal will be rejected independently of his decision).

${ }^{10}$ Our results hold for any specification of the outside value for the proposer as long as she obtains a payment less or equal to the value of her stand-alone coalition. See Section 7 in Hart and Mas-Colell [13] 
for an interpretation of a situation in which the proposer would obtain zero if the offer is rejected, and for further discussion on this extension.

${ }^{11}$ We say that a game is strictly zero-monotonic if $v(S)+v(\{i\})<v(S \cup\{i\})$ for any subsets $S \subseteq N$ with $i \notin S$ and $S \neq \Phi$.

${ }^{12}$ Again, we omit the constant $v$ and write for short $\phi_{w i}(N)$ or $P_{w}(N)$ instead of $\phi_{w i}(N, v)$ or $P_{w}(N, v)$.

${ }^{13}$ See Pérez-Castrillo [21] and Pérez-Castrillo and Wettstein [22] for the use of principals to realize cooperative outcomes.

${ }^{14}$ It can be easily shown that $\mathrm{V}(N)=\operatorname{Max}_{S \subseteq N}\{v(S)+V(N S) \mid \alpha$ in $S\}$, for any player $\alpha$ in $N$, hence when the proposer chooses the best possible coalition to be a member of, she is choosing a coalition that forms part of an efficient partition.

${ }^{15}$ Owen [20] and Hart and Kurz [10] also propose a coalition structure value to every game and every coalition structure. However, in their approach, the coalition structure serves only as a bargaining tool to increase the payoff of the members of the coalitions. At the end, all the players join the grand coalition. 